ESJ Humanities

\title{
Periurbanisation et Transport Artisanal a Yaounde (Cameroun)
}

\author{
Amagnouba Tchio Caroline
}

Chercheur, Géographe Urbaniste-INC, Cameroun

Mendouga Yannick

Chercheur, Géographe Ruraliste-INC, Cameroun

Elong Ngando Epossy Marthe Aime

Chercheur, Géographe Urbaniste-INC, Cameroun

Nguijoi Cyrille Gabriel

Chercheur, Géo-politologue-INC, Cameroun

Namekong Dagha Sinclair

Chercheur, Géo-informaticien-INC, Cameroun

Doi:10.19044/esj.2021.v17n16p40

Submitted: 23 February 2021

Accepted: 06 May 2021

Published: 31 May 2021
Copyright 2021 Author(s)

Under Creative Commons BY-NC-ND

4.0 OPEN ACCESS

Cite As:

Caroline A.T., Yannick M., Marthe Aime E.N.E., Gabriel N.C. \& Sinclair N.D. (2021). Periurbanisation et Transport Artisanal a Yaounde (Cameroun). European Scientific Journal, ESJ, 17(16), 40. https://doi.org/10.19044/esj.2021.v17n16p40

\section{Resume}

La crise économique de la fin des années 1980, a entrainé avec elle, la libéralisation des services qui, autrefois, étaient l'apanage de l'Etat, notamment le transport urbain. Cependant, la croissance démographique et l'étalement urbain spontané ont à leur tour contribué à mettre à mal le système de transport urbain au Cameroun. Cette situation a favorisé un besoin en mobilité urbaine et péri-urbaine qui reste préoccupant pour les populations dont la majeure partie d'activités socio-économiques se concentrent au centre urbain. L'inadéquation, mieux, l'absence de politiques publiques en matière de transport fait que l'Etat peine à satisfaire ce besoin, laissant entrevoir le développement d'un moyen de transport dit «artisanal » dans les villes camerounaises telles que Yaoundé. Ce travail s'appuie sur l'enquête de terrain et l'analyse des sources diachroniques et synchroniques existantes sur la ville de Yaoundé et de ses localités périurbaines. Il montre comment, malgré la présence du transport institutionnel, le «transport artisanal » est devenu depuis près de deux décennies, le principal outil de maintien de la mobilité 
centre-périphérie.

Mots clés : Périphérie urbaine, transport « artisanal », mobilité, centrepériphérie, Yaoundé

\title{
Periurbanisation and Artisanal Transport in Yaounde (Cameroon)
}

\author{
Amagnouba Tchio Caroline \\ Chercheur, Géographe Urbaniste-INC, Cameroun \\ Mendouga Yannick \\ Chercheur, Géographe Ruraliste-INC, Cameroun \\ Elong Ngando Epossy Marthe Aime \\ Chercheur, Géographe Urbaniste-INC, Cameroun \\ Nguijoi Cyrille Gabriel \\ Chercheur, Géo-politologue-INC, Cameroun \\ Namekong Dagha Sinclair \\ Chercheur, Géo-informaticien-INC, Cameroun
}

\begin{abstract}
The economic crisis of the late 1980s brought with it the liberalisation of the services that were previously the prerogative of the state, notably urban transport. However, population growth and spontaneous urban sprawl have in turn contributed to the undermining of the urban transport system in Cameroon. This situation has led to a need for urban and peri-urban mobility which remains a concern for populations whose socio-economic activities are concentrated in the urban centre. The inadequacy, or rather, the absence, of public policies on transport means that the state is struggling to meet this need, leading to the development of a so-called 'artisanal' or 'craft' means of transport in Cameroonian cities such as Yaounde. This work is based on field surveys and the analysis of existing diachronic and synchronic sources on the city of Yaounde and its peri-urban localities. It shows how, despite the presence of institutional transport, 'artisanal transport' has become, for nearly two decades, the main tool to maintain centre-periphery mobility.
\end{abstract}

Keywords: Urban periphery, "craft" transport, mobility, centre-periphery, Yaounde

\section{Introduction}

L'acception la plus communément admise de la périurbanisation en fait le processus selon lequel l'occupation quasi continue de la périphérie 
urbaine, contribue au développement des fronts d'urbanisation qui dépassent les limites des espaces couverts en infrastructures. La périurbanisation dans la ville de Yaoundé se caractérise par une extension urbaine rapide, échappant aux règles de planification des villes modernes. Dans les années 1980, la ville de Yaoundé occupait à peine $16 \%$ de son espace communal. La forte pression démographique qu'elle subit (Pape, Chenal, \& Kemajou, 2017) couplée à une planification urbaine mal maîtrisée par l'Etat (Tchékoté et al, 2015) sont à l'origine de l'extension actuelle de l'emprise urbaine, qui représentait déjà $52 \%$ en 2015 (INS, 2015). Cet étalement urbain donne progressivement naissance à des zones périurbaines de plus en plus populeuses, ce qui entraine une augmentation quasi systématique des besoins de diversification des outils de mobilité urbaine et périurbaines. Ces périphéries, comme le soulignent Motcho et Saidou (2012), ne sont pas suffisamment desservies par le transport institutionnel ${ }^{1}$ et sont moins viabilisées en termes d'infrastructures de transport. Pourtant, l'extension des couronnes périurbaines fait du secteur de l'automobile l'un des moyens de mobilité incontournables (Dominique et al., 2007).

Cette difficulté qu'éprouve l'Etat à répondre efficacement aux besoins en déplacements des populations, a laissé entrevoir, dès 1975, le développement d'un transport alternatif, dit «clandestin» par certains scientifiques (Kengne, 1985 entre autres) ou encore qualifié de «artisanal » par d'autres chercheurs (Godard, 1985; 2002 ; 2008a ; 2008b ; Fabianski, 2008 ; Chidiac, 2008 ; Boubakour, 2008 entre autres).

Ainsi, ce travail cherche à savoir comment la périurbanisation a-t-elle favorisé l'émergence du transport artisanal ${ }^{2}$ dans la métropole de Yaoundé. Quels en sont les enjeux et les perspectives ? La Réponse à ce questionnement se trouve dans l'analyse de la corrélation entre la périurbanisation spontanée et l'expansion du transport «artisanal » dans la métropole de Yaoundé (Fig. $1)$.

\footnotetext{
${ }^{1}$ C'est un mode transport instauré par l'Etat qui investit dans un service public et impose les contraintes de desserte dont, en général, les coûts ne peuvent être supportés par les usagers.

${ }^{2}$ C'est l'exploitation à une échelle individuelle de véhicules de transport public dont la propriété est atomisée, c'est-à-dire répartie entre de nombreux propriétaires. Cette exploitation peut s'intégrer dans des règles collectives plus ou moins contraignantes élaborées par des organisations professionnelles ou syndicales (Godard, 2008). Ces dernières définissent les voies de dessertes, payent les taxes et fixent les coûts à l'unanimité avec les autorités administratives.
} 
European Scientific Journal, ESJ

May 2021 edition Vol.17, No.16

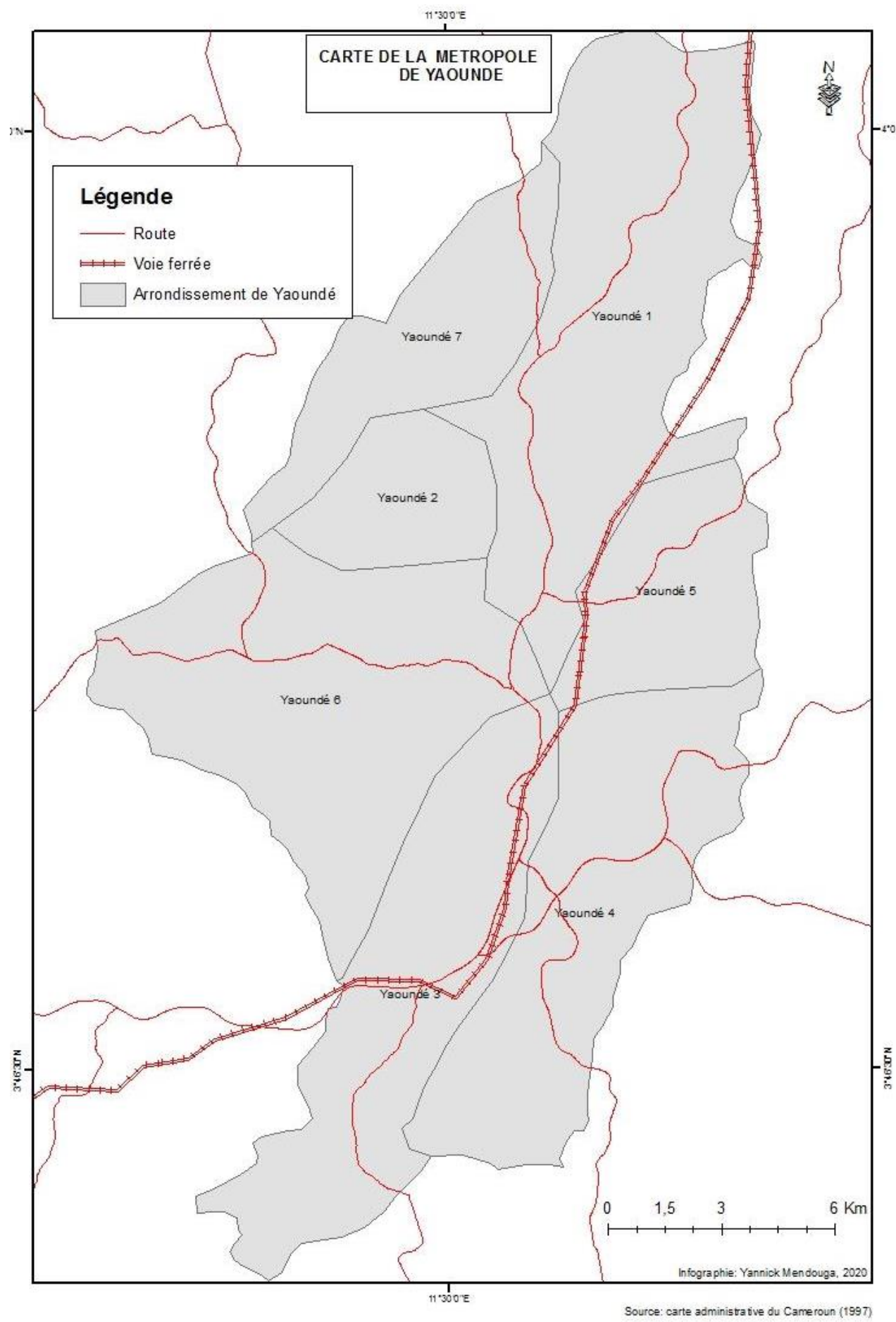

Fig.1. Métropole de Yaoundé 


\section{Methodologie}

La méthodologie de recherche utilisée dans le cadre de cette étude est essentiellement analytique et permet de faire une synthèse transdisciplinaire des phénomènes de la périurbanisation et le développement du transport dit «artisanal » dans la métropole de Yaoundé. Cette étude est fondée sur : des observations de terrain, la recherche documentaire, et les entretiens menés non seulement, auprès des gérants de stationnement du transport artisanal desservant la périphérie urbaine et des usagers rencontrés sur les points de ramassage. Elle s'est aussi appuyée sur les informations reçues des acteurs institutionnels en charge du transport au Cameroun. Ces données sont donc catégorisées en données primaires (empiriques) collectées en mai 2020 et secondaires.

Les données empiriques sont le fruit des interviews semi-directives auprès des 07 gérants de stationnement ; 100 passagers sélectionnés au hasard, soit plus d'une dizaine par site d'embarquement ; et auprès des responsables du ministère du transport notamment le Chef service du transport urbain et périurbain.

Le questionnaire destiné aux gérants de stationnements visait à comprendre le fonctionnement global de l'activité. Ces entrevues ont permis de situer le contexte économique et spatial dans lequel s'effectue la mobilité périurbaine, ainsi que ses enjeux. Celui destiné aux usagers (passagers) permet d'analyser les logiques de choix de chaque acteur dans leurs mouvements pendulaires. Ces entrevues ont aussi donné lieu aux observations et aux levées cartographiques afin de compléter la base de données.

Les données secondaires proviennent essentiellement de la revue de la littérature, de l'exploitation des données issue du Ministère des transports et de la Communauté Urbaine (CU). Le traitement des données collectées a été fait à l'aide d'Excel, ainsi que les logiciels de traitement d'image pour la réalisation des cartes illustrant cette étude notamment, ArcGis 10.3.1 et QGis 2.12.1.

\section{Periurbanisation et Enjeux Sur la Mobilite a Yaounde}

Lors de son extension spatiale dans les années 1980, le noyau urbain de la ville de Yaoundé est constitué d'environ 38,7 km2 soit 3807ha (Tchékoté, Kaffo, 2012 : 98). Mais, du fait d'une urbanisation rapide marquée par le développement systématique des blocs périurbains allant au-delà des limites de la zone couverte en infrastructures de transport, ce noyau est passé à pratiquement 30.000 ha en 2015 (Mfoulou Olugu, 2016 :2) la mobilité urbaine devient désormais un véritable défi des pouvoirs publiques. Malgré les systèmes de mobilités institutionnelles mis sur pied à partir des années 1973 la périurbanisation spontanée met à mal le cours de ce projet ainsi que les 
effets escomptés. En conséquence, par l'augmentation des besoins de mobilité, on assiste à la montée d'une régulation autonomique du transport.

\section{Le périurbain de la ville de Yaoundé : une dynamique spatiale mal maitrisée}

La dynamique urbaine reste très complexe en ce sens qu'elle est fortement liée aux soubresauts du temps puisque fondée au début de la pénétration coloniale, d'abord Allemande dès 1889, puis française dès 1916, et structurée par des contingences socio-culturelles et politico-économiques qui ont rythmé l'évolution du Cameroun ces quatre dernières décennies (Voundi, Tsopbeng, et Tchindjang, 2018 ; Bopda, 1984 ; Youana, 1980). La configuration spatio-temporelle du paysage urbain de Yaoundé est fortement marquée par l'extension rapide du noyau urbain vers ses périphéries autrefois rurales, mettant à l'épreuve les outils de planification développés par l'État.

Au début des années 1980, le tissu urbain de Yaoundé était très peu peuplé selon René Joly Assako Assako (2011). Mais de nos jours, il connait un développement rapide, avec une superficie sans cesse croissante (Fig. 2), favorisé par une croissance démographique annuelle de $2.37 \%$, laissant libre cours à des installations anarchiques (Tchekote \& Ngouanet, 2015, p 264). 
Fig. 2. Evolution du tissu urbain de Yaoundé entre 1980 et 2001

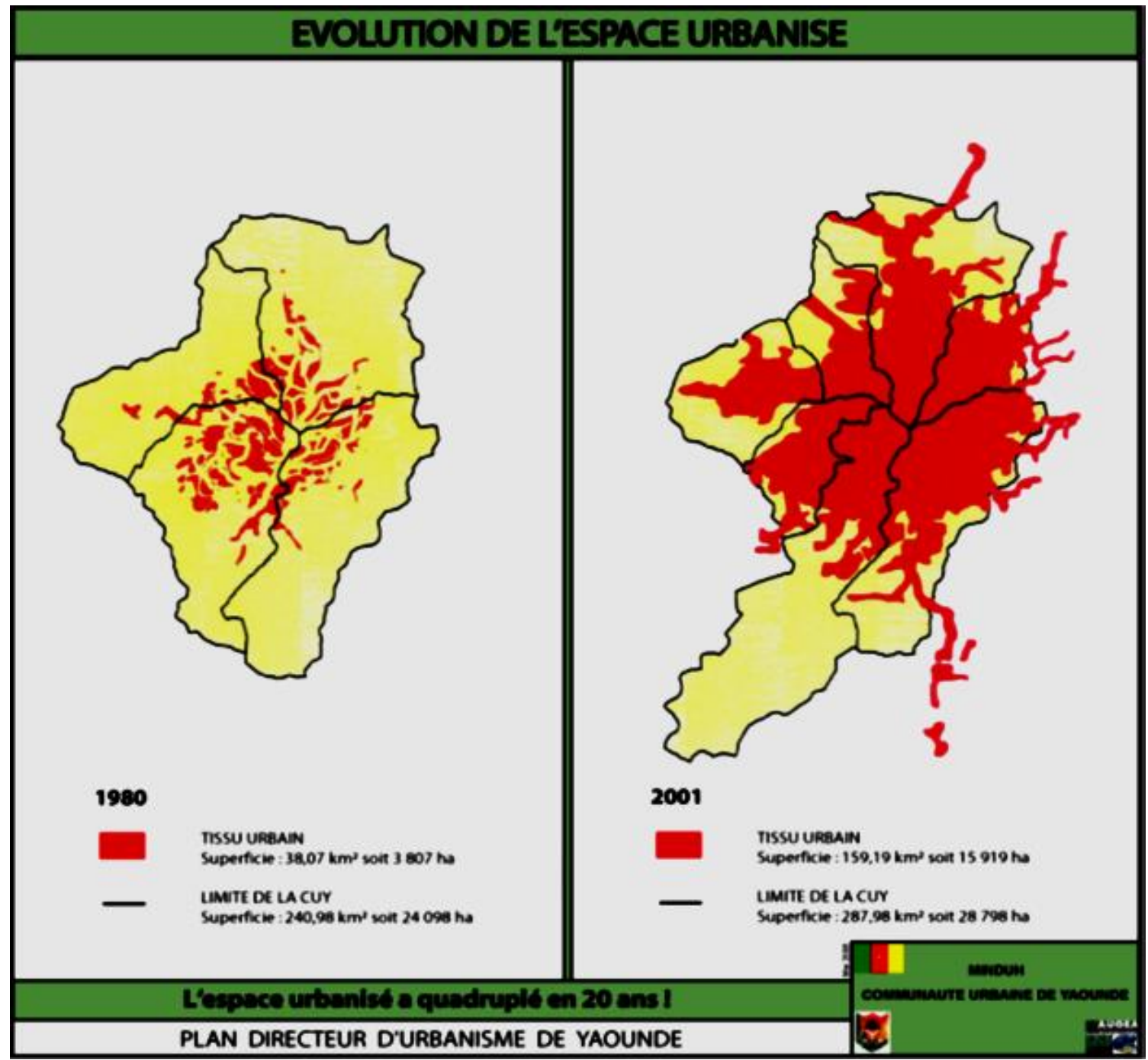

Source : Communauté Urbaine de Yaoundé (CUY), recherche documentaire, Juin 2020

Cet étalement urbain s'est accentué au début des années 2000, bien que certaines données montrent en effet que la croissance fulgurante de la population dans ces espaces, se manifeste beaucoup plus entre 2010 et 2018. Elle est en constance évolution (Tableau 1), due en grande partie à l'accès facile à la propriété foncière dans les périphéries de la ville, en complicité avec les autorités administratives et traditionnelles (Assako Assako \& Njouonang, 2016, p 19). 
Tableau 1. Croissance démographique à Yaoundé

\begin{tabular}{|c|c|c|}
\hline Population/ Estimation & Urbaine & \multirow{2}{*}{ Taux d'urbanisation } \\
\hline 2001 & Evaluée à 1,5 millions d'habitants & \multirow{2}{*}{ Passé de $48,5 \%$ à $71 \%$} \\
\hline 2010 & Evaluée à 2,19 millions & \\
\hline 2020 & Evaluée à 3,32 millions & \\
\hline 2035 & 5,6 millions & \\
\hline
\end{tabular}

Source : Données PDU (2010-2020)

La ville de Yaoundé connait une forte métropolisation depuis le début des années 2000 (Ongolo, 2002; CUY, 2010; Mfoulou Olugu, 2017). Aujourd'hui, l'extension de la ville se poursuit de manière non planifiée, où naissent de nombreux quartiers dans lesquels les habitants occupent des espaces moins coûteux. Cette extension a pour principales causes : une saturation des espaces centraux disponibles entrainant un éclatement et un desserrement de l'emprise urbaine ; une offre abondante et bon marché de terrains en périphéries urbaine.

Le périurbain s'étend actuellement sur $60 \%$ de la superficie de la ville et abrite près de $70 \%$ de la population (Voundi, Tsopbeng, et Tchindjang, 2018). Ceci dit, la métropole de Yaoundé couvre une superficie estimée, en 2010, à environ $310 \mathrm{~km}^{2}$, soit 28.798 hectares dont $44 \%$ représentent la surface périurbaine (CUY, 2010). Le taux de croissance du périmètre urbanisé moyen est estimé à 9,1\% (Op. Cit.). Les tendances récentes indiquent une croissance urbaine sur 04 fronts notamment au Nord/Nord-Est (RN1 et route Soa), à l'Est (RN10 et route de Mfou), au Sud (route de Nsimalen) et au Sud-Ouest (vers l'extension de Simbock) (Plan d'Actions, 2019, p. 22).

Il s'agit en règle générale, d'une extension qui s'étale vers la sortie de la ville, limitée par le relief relativement inaccessible du côté d'Oyomabang, Nkolbisson et Leboudi. Ces quartiers sont en cours de densification, malgré l'existence en majorité des terres peu constructibles notamment les marécages et les collines etc. En même temps, d'autres quartiers se densifient et se consolident rapidement. Des études ((Tchékoté \& Ngouanet, 2015, p 264 ; Ongolo, 2002 ; CUY, 2010 ; Mfoulou Olugu, 2017 ; CUY, 2010) montrent ainsi une excroissance spontanée vers les sorties Est, malgré l'existence des rivières Anga'a et Foulou, et Sud-Ouest du côté d'Afanoyoa. Cette extension urbaine accentue les besoins en termes de mobilité urbaine, qui influe sur le système de transport disponible pour satisfaire cette demande.

\section{Impacts du périurbain sur la mobilité urbaine}

L'accélération du phénomène de périurbanisation observé ces dernières années à Yaoundé impact la mobilité urbaine de manière significative. Selon le Plan Directeur d'Urbanisme (PDU) de 2020, la population de Yaoundé est passée de 1,81millions d'habitants en 2005, à 3,32millions en 2020. En appliquant les hypothèses de croissance du Plan de 
Mobilité Urbaine de 2019, la population de Yaoundé atteindrait 5,6 millions d'habitants en 2035. Cette croissance démographique dont le taux moyen, depuis le dernier recensement (2005) est estimé à 3,1\%, met en scène au moins deux niveaux d'impacts. D'abord, un accroissement des mobilités urbaines de longue distance ; et ensuite, une limitation de la capacité structurelle du transport institutionnel à desservir toutes les artères urbaines et périurbaines.

Le couple étalement urbain et croissance démographique (Ndzana, Etoundi Tsala, \& Ongolo Zogo, 2012), a significativement influé sur l'augmentation des besoins en mobilité (tableau 2) du fait accroissement du trafic depuis 2010.

Tableau 2. Aperçu de l'évolution des besoins en mobilité urbaine à Yaoundé

\begin{tabular}{|c|c|c|c|}
\hline Année & Offre & Demande & Projection \\
\hline $\begin{array}{l}2010- \\
2018\end{array}$ & $\begin{array}{l}190000 \\
\text { véhicules } \\
\text { automobiles, soit } \\
75 \text { bus }\end{array}$ & $\begin{array}{l}\text { De } 12000 \text { mouvements par jour, à près de } \\
500 \text { 000/jour soit } 64 \% \text { vers les périphéries }\end{array}$ & \\
\hline $\begin{array}{l}\text { horizons } \\
2020- \\
2035\end{array}$ & & $\begin{array}{l}854000 \text { mouvements de véhicules/jour } \\
\text { Représentant } \\
5 \text { millions de déplacements/jour, Soit une } \\
\text { augmentation de } 10 \%\end{array}$ & $\begin{array}{l}120000 \text { mouvements de } \\
\text { véhicules, soit } 1,7 \\
\text { million } \\
\text { mouvements/jour } \\
4,2 \quad \text { millions de } \\
\text { déplacements par jour }\end{array}$ \\
\hline
\end{tabular}

Sources : données PDU (2010), tableau réalisé par les auteurs 2020.

La croissance démographique est l'un des principaux facteurs de l'expansion de la structure urbaine de Yaoundé, avec pour conséquence une augmentation du trafic quotidien. Comme l'indique le tableau 2, l'offre en matière d'outil de transport urbain est estimée à 190.000 taxis et 75 bus dans la période 2010-2018. Pourtant, dans la même période, la demande s'élevait déjà à 12.000 Mouvements par jour, dont $64 \%$ vers les périphéries. Ce qui était déjà assez limite, puisque les projections situent la demande en termes de mobilité à environ 854.000 mouvements de véhicules par jour entre 2020 et 2035, ce qui représenterait 5 millions de déplacements par jour, soit une augmentation de $10 \%$.

La forte demande en mobilité a mené à une réorganisation du service de transport public par les acteurs privés, dans l'optique d'être plus attractive et plus efficace, et cela n'a été possible qu'à travers la création des pôles de concentration urbaine où le transport artisanal a pu se développer.

Au regard de la croissance du trafic en milieu urbain, l'insuffisance de l'offre de transport et l'accès limité dans les nouveaux quartiers affectent positivement les choix de mobilité vers le transport artisanal avec une probabilité oscillant autour de $86 \%$. En réalité, le choix du transport artisanal dépend de plusieurs facteurs, parmi lesquels, l'accessibilité. Le transport artisanal se trouve accessible et se positionne donc au cœur de la relation 
périurbanisation-transport. Ce moyen de transport favorise aussi bien la localisation des activités urbaines, périurbaines et les modes de mobilité, que l'utilité du choix de mobilité. L'accessibilité au transport artisanal influence l'usage des minibus et des voitures particulières, comme moyen alternatif au déficit du transport institutionnel.

\section{Emergence du Transport Artisanal : Une Solution Alternative Pour la Mobilite Urbaine}

L'Etat et ses partenaires (Banque Africaine de Développement, Banque Mondiale) fournissent des efforts permanents (depuis 1973) pour mettre en place un système transport collectif (Ngabmen, Broutin, \& Essomba Eloundou, 2001). Ce système de transport face à une demande très supérieure à l'offre du fait de l'extension spatiale très prononcée de la métropole de Yaoundé. Ainsi, cet état de choses favorise l'émergence du transport dit « artisanal » sous l'égide des particuliers.

\section{De la périurbanisation à l'émergence du transport artisanal : Quand les transporteurs particuliers prennent en main le service du transport urbain et périurbain}

La forte pression démographique (voir tableau 1) favorise l'émergence du transport artisanal qui vient en appui au problème de mobilité des personnes du centre vers les périphéries. Les quartiers périphériques à la ville de Yaoundé et des arrondissements voisins (Soa, Obala, Okola, Mbankomo, Nkolafamba, Mfou etc.) sont sans cesse croissants augmentant ainsi les demandes de déplacement du centre urbain vers les périphéries et vice-versa. La concentration des points d'embarquement dans le centre urbain (Fig. 3) s'explique par le fait qu'il concentre la majeure partie des sites d'activités socio-économique, administratives et scolaires de la métropole de Yaoundé. C'est état de choses est responsable de 50\% des déplacements urbains. 
European Scientific Journal, ESJ

May 2021 edition Vol.17, No.16

Fig. 3. Emprise spatiale du transport par autocar et minibus à Yaoundé

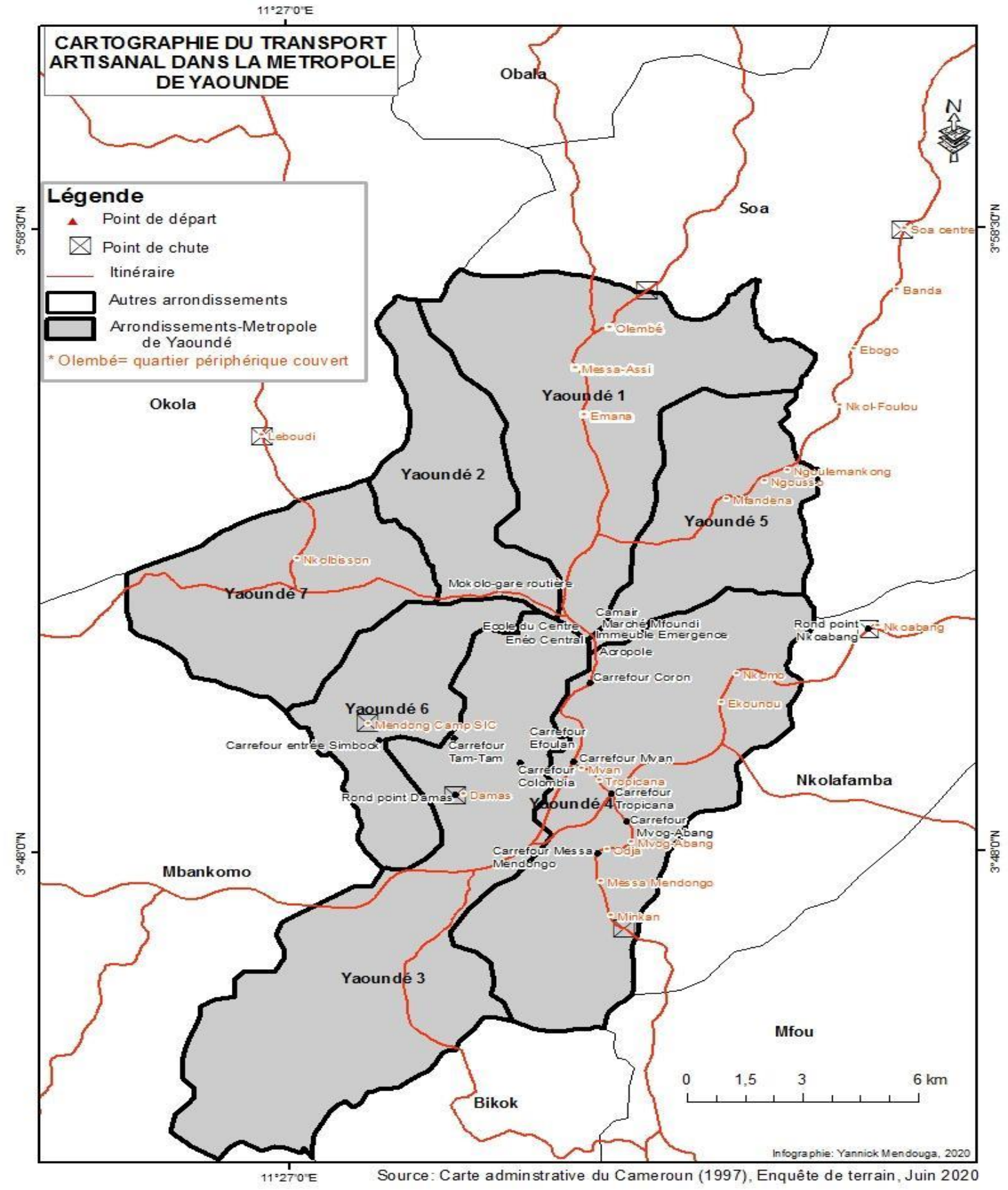




\section{Transport artisanal : un moyen incontournable pour la mobilité urbaine et périurbaine dans la métropole de Yaoundé de nos jours}

A Yaoundé, le transport artisanal en général assure près de 53\% des déplacements contre 3\% assurés par le transport institutionnel (MobiliseYourCity, 2019, p.10). Dans ces proportions, les autocars et les minibus (Planche 1) qui assurent le transport des personnes entre le centre urbain et les périphéries, couvrent 3\% du transport urbain.

Planche 1. Exemple d'aire de stationnement de minibus au lieu-dit Camair
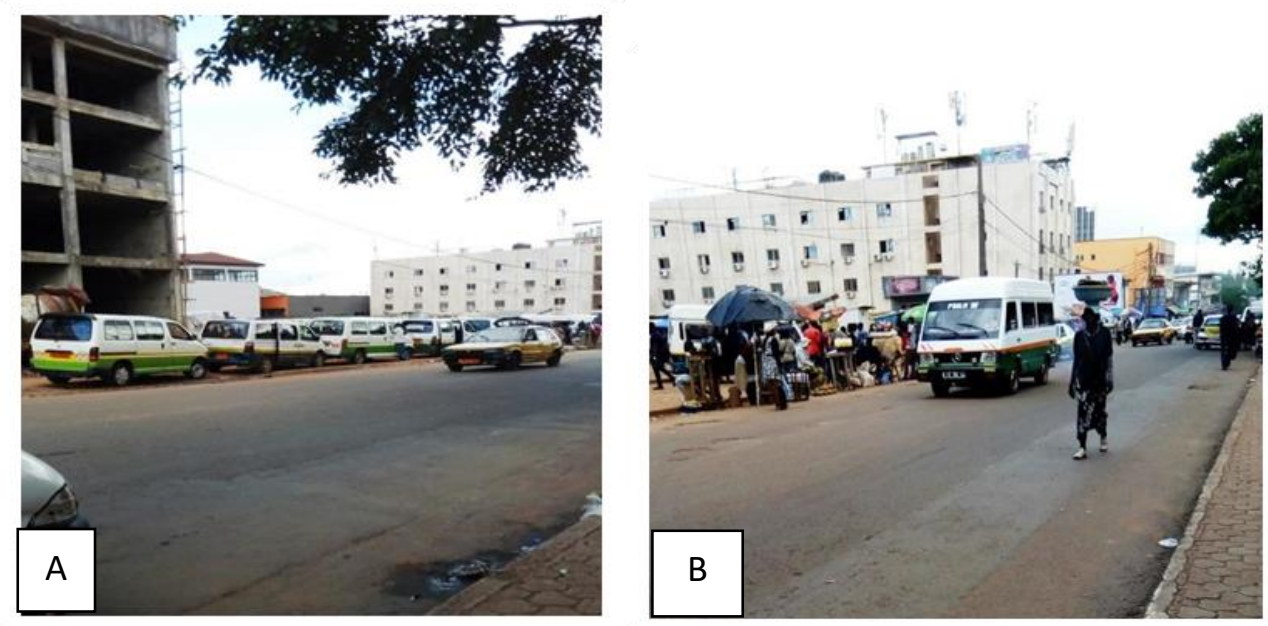

Source. Auteurs, Enquête de terrain, mai 2020

Le transport par autocar et Minibus : un moyen d'accès rapide à la périphérie urbaine

L'incapacité de l'Etat à mettre sur pieds les moyens de transport en quantité suffisante estimés à 3 bus pour 100000 habitants, et les infrastructures y relatives est une aubaine pour le développement du transport artisanal. Cela se justifie par le fait que le transport artisanal parvient plus ou moins à satisfaire les besoins de mobilité urbaine et périurbaine dans la métropole de Yaoundé.

L'émergence du transport artisanal par autocar et Minibus date des années 1975 (Kengne F., 1985), période où la périurbanisation commence à se ressentir de façon considérable. Excepté la station de Camair qui dessert la zone de Soa (créée en 1994, après l'ouverture de l'université de Soa), les autres points d'embarquement sont le fruit d'une demande importante de mobilités des personnes du centre vers les périphéries, et vice-versa. 
Fig. 4. Dates de création des différents stationnements de transport par autocar et Minibus

\begin{tabular}{|c|c|c|c|c|c|c|}
\hline \multirow{5}{*}{$\begin{array}{l}2020 \\
2010 \\
2000 \\
1990 \\
1980 \\
1970\end{array}$} & & & & & & 2013 \\
\hline & 1990 & 1994 & 1994 & 1995 & 2000 & \\
\hline & & & & & & \\
\hline & & & & & & \\
\hline & 离 & 芯 & 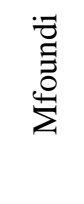 & $\begin{array}{l}\frac{0}{0} \\
0 \\
0 \\
0 \\
\frac{0}{4}\end{array}$ & 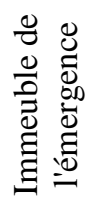 & 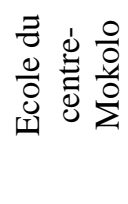 \\
\hline
\end{tabular}

Source. Enquête de terrain Juin, 2020

Dans l'optique de satisfaire les demandes de mobilités centrepériphérie, les particuliers mettent à la disposition des usagers des véhicules d'une capacité comprise entre 05 et 30 places assises à partir de leurs fonds propres. Les investigations menées sur le terrain nous ont permis d'identifier au total 490 véhicules impliqués dans ce type de transport (Tableau 3).

Tableau 3. Nombre de véhicules par points d'embarquements

\begin{tabular}{|l|l|l|}
\hline Points d'embarquements & Nombre de véhicules & Propriétaires \\
\hline Enéo Centrale & 40 & Particuliers \\
\hline Camair, Mfoundi & 300 & Particuliers \\
\hline Immeuble émergence & 50 & Particuliers \\
\hline Ecole du centre-Mokolo & 50 & Particuliers \\
\hline Acropole & 50 & Particuliers \\
\hline TOTAL & 490 & \\
\hline
\end{tabular}

Source. Enquête de terrain, Juin 2020

Malgré l'état voire l'étroitesse des voies de communication partant du centre urbain vers les périphéries, les transporteurs réussissent l'exploit de desservir ces zones à moindres coûts fixes (Tableau 4) par rapport aux taxis de ville. Etant donné que la population vivant à la périphérie du centre urbain est plus ou moins nantie, elles font du transport par minibus et autocars le leader des transports en zone périphérique de nos jours. 
Tableau 4. Coûts fixes du transport artisanal à Yaoundé

\begin{tabular}{|c|c|c|}
\hline Départ & Arrivée & Coûts fixes de transport (en FCFA) \\
\hline \multirow{2}{*}{ Enéo centrale } & Mendong & 200 \\
\hline & Damas & 200 \\
\hline \multirow{5}{*}{ Camair } & Mobil Omnisport & \multirow{5}{*}{250} \\
\hline & Ngousso & \\
\hline & Eleveur & \\
\hline & Nkoulemekong & \\
\hline & Soa & \\
\hline \multirow{3}{*}{ Mfoundi } & Emana & \multirow{3}{*}{300} \\
\hline & Messa-Assi & \\
\hline & Olembé & \\
\hline \multirow{3}{*}{ Acropole } & Ekounou & 150 \\
\hline & Nkomo & 200 \\
\hline & Nkoabang & 250 \\
\hline \multirow{2}{*}{$\begin{array}{c}\text { Ecole du } \\
\text { centre-Mokolo }\end{array}$} & NKolbison & \multirow{2}{*}{250} \\
\hline & Leboudi & \\
\hline \multirow{5}{*}{$\begin{array}{l}\text { Immeuble } \\
\text { Emergence }\end{array}$} & Mvan & \multirow{3}{*}{250} \\
\hline & Odja & \\
\hline & Messamendongo & \\
\hline & Nkolnda & 300 \\
\hline & Minkan & 500 \\
\hline
\end{tabular}

Source. Enquête de terrain, Juin 2020

L'enquête menée auprès de 100 usagers nous a révélé qu'il regorge d'énormes avantages pour la population installée dans les quartiers périphériques : sa disponibilité, sa rapidité, son coût relativement bas par rapport aux taxis, sa proximité et sa sécurité. Ce dernier avantage sous-entend l'absence d'agression comme dans les taxis de ville et le faible taux de perte des bagages. Cela justifie pourquoi ce moyen de transport rafle quasiment la clientèle des taxis de ville et amène certains propriétaires de voitures à l'adopter dans le but d'échapper aux congestions et aux dépenses en carburant que cela engendre. Par exemple, sur les 70 usagers ayant été interrogés, 11 déclarent que bien avant les autocars et minibus, ils se déplaçaient avec leurs propres véhicules ; 27 empruntaient le taxi de ville ; seuls 32, constitués majoritairement de personnes en quête d'un loyer abordable, l'empruntent exclusivement depuis leur installation à la périphérie.

\section{Transport dit « artisanal » : Un service de transport sous la vigilance des acteurs institutionnels et particuliers ?}

Le transport dit «artisanal » dans les villes camerounaises en général et dans celle de Yaoundé en particulier, bien que pratiqué par des particuliers, est accompagné par les institutions en charge des questions de transport, selon le niveau d'intervention. Les acteurs institutionnels qui interviennent dans le 
suivi de cette pratique en milieux urbains et périurbains sont constitués entre autres de :

- Ministère du transport et ses services déconcentrés: à travers des arrêtés $^{3}$ réglementant le service de transport en général et celui « artisanal» en particulier, le Ministère du Transport (MINT) est succinctement chargé de délivrer l'agrément, les licences spéciales S1 et $\mathrm{S} 4$, de définir la classe de véhicule et le nombre de places, de vérifier l'état des véhicules, de définir avec la commune territorialement compétente les points d'embarquement et de déchargement avec pour base le centre urbain ;

- Ministère du Commerce : Il est chargé d'homologuer les prix du transport tant urbain que périurbain ;

- Communes et Communauté Urbaine : le décret N²015/4209/PM du 24 Novembre 2015 fixant les modalités d'exercice de certaines compétence transférées par l'Etat aux communes ou aux communautés urbaines en matière d'organisation et de gestion des transports publics urbains, fait des communes et les communautés urbaines notamment de la métropole de Yaoundé des acteurs incontournables du service de transport notamment celui « artisanal».

La communauté urbaine et les sept (7) communes de la ville de Yaoundé sont succinctement chargées de la création et de la gestion des infrastructures de transport (depuis 2010) ${ }^{4}$, de l'élaboration du plan de déplacements urbains et périurbains, et de la délivrance des licences et des cartes bleues. Cependant, cette vigilance à elle seule ne suffit pas pour vaincre le problème de mobilité urbaine.

\section{Discussion}

Le processus de périurbanisation et le développement des moyens de transport sont vus comme étant liés par une causalité circulaire dans les pays développés (Laugier, 2012). Ces études ont démontré que le développement des infrastructures de transport a fortement contribué à l'accroissement du processus de la périurbanisation. Certains auteurs, à l'instar de Wiel M.

${ }^{3}$ (1) $\mathrm{N}^{\circ} 0000182 / \mathrm{MINT}$ du 16 février 2006 fixant les conditions et modalités d'exploitation, à titre onéreux, du service de transport urbain par autocar ou autobus ; (2) $\mathrm{N}^{\circ} 0000184 / \mathrm{MINT}$ du 16 février 2006 fixant les conditions et modalités d'exploitation, à titre onéreux, du service de transport urbain et péri-urbain de personnes ; (3) $\mathrm{N}^{\circ} 00003 / \mathrm{MINT}$ du 05 Janvier 2017 fixant les modalités d'exploitation, à titre onéreux, du service du transport urbain, périurbain et rural de personnes par minibus.

${ }^{4}$ Il s'agit d'une compétence qui a été transférée aux 360 communes du pays par le Ministère des Travaux Publics depuis 2010. Ce transfert prévoit aussi un accompagnement financier annuel par ledit ministère dans le but de leur permettre d'assurer pleinement cette compétence. 
(2005), identifient l'impact des modifications des technologies de transport sur les formes urbaines, en ce sens que, les différences de vitesses entre l'automobile et la marche à pieds ont conduit à un éloignement de l'espace constructible pour un même temps d'accès au travail. Pour lui, «l'amélioration des temps d'accès par les infrastructures routières a induit une valorisation sélective de l'espace, ce qui a provoqué le redéploiement des fonctions urbaines. La mobilité facilitée a donc libéré la ville de la «tyrannie » de la distance avec ses effets sur la densité urbaine » (Wiel, 1999, p89). Il précise que l'étalement urbain s'explique en partie par la généralisation des vitesses de déplacement accrues favorisées par la création d'infrastructures routières. En outre, le coût généralisé de transport, défini comme la somme du coût monétaire direct (voiture, essence, entretien) et du coût du temps de transport, a fortement baissé, entraînant ainsi l'extension périurbaine des villes.

Cependant, ce travail tend à ressortir une réalité autre que celle présentée plus haut. Les investigations de terrain révèlent, tel que présenté dans la figure 4, que l'émergence et le développement des moyens de transports artisanaux intervient à la suite de l'accentuation de la périurbanisation dans la métropole de Yaoundé. Cette accentuation est surtout le fruit d'une quête perpétuelle de terres et de loyers relativement bas en périphéries. En prenant l'exemple de quelques zones périphériques de la ville de Yaoundé comme Soa, Nkoabang, Nkolmesseng, Dagobert, Symbock et autre, l'on se rend compte que la population s'est d'abord installée et c'est quelques années après que les infrastructures et les moyens de transport ont suivis.

Il est cependant important de souligner que cet étalement urbain est la cause de plusieurs raisons. Pour des familles, la qualité de vie est sans conteste meilleure en périphérie : le cadre de vie offre de la diversité, les logements sont plus spacieux, des prix immobiliers moins élevés, le bruit et la pollution sont atténués, la nature est plus accessible, et il est plus facile de lier connaissance avec ses voisins qu'en ville (le manque d'habitants permet de mieux se rencontrer : tout le monde fréquente les mêmes commerces, les mêmes évènements organisés par la collectivité). Dans la ville de Yaoundé, l'accessibilité aux moyens de transport et les densités humaines ont une forte probabilité ( $82 \%$ et $63 \%$ respectivement) d'influencer le choix de mobilité en faveur du transport de masse. Les variables de forme urbaine et les caractéristiques socio-économiques de la zone d'origine ont respectivement un impact sur le coût de mobilité quel que soit le moyen de déplacement. Ces résultats militent en faveur d'un réaménagement des zones urbaines, l'objectif étant de réguler l'étalement urbain, de faciliter l'accessibilité aux transports collectifs et d'accroître l'efficacité et la compétitivité du transport de masse afin de réduire la part usagère du transport artisanal. 


\section{Conclusion}

Le but de ce travail était d'analyser comment la périurbanisation a contribué au développement du transport artisanal dans la métropole de Yaoundé. Il consistait également à questionner de manière implicite le problème de la gouvernance du transport urbain au Cameroun. Au terme de cette étude, il en ressort que le périurbain s'est élargie de façon anarchique, sous la pression démographique. La relation centre-périphérie à travers la concentration des activités au centre urbain trouve ici sa place et laisse donc entrevoir le développement du transport artisanal notamment, par autocar et minibus comme solution au problème de mobilités. Ce travail s'inscrit également dans le champ des différentes réflexions sur les jeux, les enjeux et les contradictions qui existent dans les politiques d'aménagement urbain aussi bien au Cameroun, que dans la plupart des pays d'Afrique au Sud du Sahara. Il est toutefois singulier en ce sens qu'il relève le rôle des dynamiques spatiotemporelles, et démographiques, ainsi que leur implication, tout en mobilisant les outils cartographiques pour inscrire le périurbain de Yaoundé dans une démarche qui interpelle les acteurs aussi bien institutionnels que noninstitutionnels, et qui participent de manière étroite, à la construction des espaces.

\section{References:}

1. ASSAKO ASSAKO, R. (. (2011). Yaoundé. La métropole face à son arrière-pays. Paris: Éditions Dianoïa, 176 p.

2. ASSAKO ASSAKO, René Joly \& NJOUONANG, Djomo. (2016). Etalement urbain et insecurite fonciere dans la banlieue nord de yaounde : prolegomenes d'un modele de gestion integree du sol en milieu perimetropolitain africain. ResearchGate, https://www.researchgate.net/publication/314238633, pp.15-40.

3. BOPDA, A. (1984). Dynamique de l'espace urbain de Yaoundé, reconstruction et expansion postcoloniale du bâti. Thèse de 3ème cycle, Université de Yaoundé, $317 \mathrm{p}$.

4. BOUBAKOUR, F. (2008). Transport artisanal et transport par taxis clandestin. Dans X. Godard, Le transport artisanal dans les villes méditéranéennes (pp. 61-77). Actes INRETS N¹14.

5. CHIDIAC, R. (2008). Le transport artisanal dans un contexte de crise politique: le cas du Liban. Dans X. Godard, Le transport artisanal dans les villes méditéranéennes (pp. 41-60). Actes INRETS N¹14.

6. CUY. (2010). Elaboration du plan de déplacement Urbain de la ville de Yaoundé. Yaoundé: Rapport Diagnostique, République du Cameroun. 
7. CUY. (2020). PLAN DIRECTEUR D'URBANISME. Yaoundé: Rapport de Présentation (MINISTERE DU DEVELOPPEMENT URBAIN ET DE L'HABITAT).

8. FABIANSKI, C. (2008). Le transport privé dans le cas d'Istambul. Dans X. Godard, Le transport artisanal dans les villes méditéranéennes (pp. 27-40). Actes INRETS $\mathrm{N}^{\circ} 114$.

9. GODARD, X. (1985). Quel modèle de transports collectifs pour les villes africaines? (Cas de Brazzaville et Kinshasa). Politique africaine $N^{\circ} 17,41-57$.

10. GODARD, X. (2002). Les transports et la ville en Afrique au Sud du Sahara: le temps de la débrouille inventif. Paris: Krthala.

11. GODARD, X. (2008). Introduction à la question du transport artisanal en Méditérranée. Le transport artisanal dans les villes méditérranéenes : Compte-rendu du Séminaire de Recherche du 5-6 jin 2007 (pp. 11-21). Aix en Provence: Maison Méditérranéenne des Sciences de l'Homme, Actes INREST $\mathrm{N}^{\circ} 114$.

12. GODARD, X. (2008). Transport artisanal, esquisse de bilan pour la mobilité durable. Codatu XIII. Hô Chi Minh Ville (Vietnam), 10.

13. KENGNE, F. F. (1985). Les transports "clandestins" autours de Yaoundé. Cahiers d'Outres-mers, 175-195.

14. LAUGIER, R. (2012). L'étalement urbain en France : Synthèse documentaire. Paris, 23P.: CRDALN.

15. MFOULOU OLUGU, J. P. (2017). Dysfonctionnements de l'offre de transport en Afrique: cas de Yaoundé. Groupe de la Banque Africaine de développement, ocument de travail $n^{\circ} 262,37 \mathrm{p}$.

16. MOBILISEYOURCITY. (2019). République du Cameroun : Politique nationale de la mobilité urbaine - Diagnostic, vision nationale et mesures stratégiques pour sa réalisation. $93 \mathrm{p}$

17. MOTCHO, K. H., \& Saidou, H. Y. (2012). Grandeur et décadence des taxis banlieue Talladjé-talladjé de la ville de Niamey. EspaceS Temps. net.

18. MOUTILA BENI, L. (2013). PLANIFICATION URBAINE AU CAMEROUN:NATURE, ORIGINE, ET DEFIS. Consulté le Juin 13, 2020, sur Géographie et environnement: https://moutilageo.hypotheses.org/17

19. NDZANA, Arnauld Philippe, ETOUNDI TSALA, Louis Freddy \& ONGOLO ZOGO,Valérie. (2012). La mise en œuvre du Plan de Déplacements Urbains de Yaoundé : enjeux, contraintes et méthodes. Conférence CODATU XV sur: "Le rôle de la Mobilité Urbaine pour (re)modeler les villes », 22-25 Octobre, (p. p.18). Addis Abeba (Ethiopie). 
20. NGABMEN, H., BROUTIN, C., \& ESSOMBA ELOUNDOU, A. (2001). Libéralisation de l'exploitation des transports collectifs urbains à Douala et à Yaoundé: Chronique de la mise en oeuvre d'une nouvelle approche. Accra: Banque Mondiale.

21. ONGOLO, Z. V. (2002). Quel modèle de concession des réseaux de transport dans les capitalesafricaines: le cas de Yaoundé. CODATU, Lomé, A ABalkema, pp. 309-313.

22. PAPE, S., CHENAL, J., \& KEMAJOU, A. (2017). Villes africaines: Mobilités et transports urbains. 1st Edition, 60p.

23. Plan d'Actions. (2019). Plan d'actions du Plan de Mobilité Urbaine Soutenable pour la Communauté Urbaine de Yaoundé. Yaoundé: Mobilise Your City.

24. TCHEKOTE, Hervé \& NGOUANET, Chrétien. (2015). Périurbanisation anarchique et problématique de l'aménagement du territoiredans le périurbain de Yaoundé.

ResearchGate,https://www.researchgate.net/publication/312916710, pp.259-270.

25. VOUNDI, Eric TSOPBENG, Carole et TCHINDJANG, Mesmin . (2018). Restructuration urbaine et recomposition paysagère dans la ville de Yaoundé. VertigO- la revue électronique en sciences de l'environnement, Volume $18 N^{\circ} 3$, [en ligne].

26. WIEL, M. (1999). La transition urbaine: Le passage de la ville pédestre à la ville motorisée. MARDAGA, Collection Recherches, $149 \mathrm{P}$.

27. WIEL, M. (2005). Ville et mobilité: un couple infernal? Paris: Editions de l'aube. La tour d'Aigues.

28. YOUANA, J. (1980). Les quartiers spontanés péricentraux de Yaoundé. Une contribution à l'étude des problèmes de l'habitat du plus grand nombre. Thèse de Doctorat de 3e cycle, Université de Yaoundé, $324 \mathrm{p}$. 